\title{
La inclusión, un derecho humano no garantizado aún en la universidad autónoma de baja california
}

\section{Inclusion As Human Right Is Not Assured Yet at the Autonomous University of Baja California}

Carmen Amalia Plazola Rivera Universidad Autónoma de Baja California

Baja California, México carmen.plazola@uabc.edu.mx

Víctor Hugo Saldaña Guevara Universidad Autónoma de Baja California

Baja California, México victor.saldana@uabc.edu.mx

Agustín Manuel Velázquez Bustamante Universidad Autónoma de Baja California

Baja California, México amvb_1959@uabc.edu.mx

Sixto Luna Cruz Universidad Autónoma de Baja California Baja California, México sixtolunacruz@uabc.edu.mx

Recibido: 16/05/2018 Aceptado: 04/09/2018

Resumen. Si bien es cierto que en nuestra Universidad Autónoma de Baja California se han esforzado en pretender lograr la inclusión, esta no se ha logrado.

Hablamos de la inclusión de las personas con alguna discapacidad, las cuales, a pesar de ser aceptadas por dicha institución, no han logrado la garantía de esa inclusión, violentando con ello lo previsto por nuestra Constitución en su artículo primero, párrafo último, que refiere: "Queda prohibida toda discriminación motivada por origen étnico o nacional, el género, la edad, las discapacidades, la condición social, 
ReVISTA Universidad EN DiÁlogo • Vol. 9, N. ${ }^{\circ}$ 1, Enero-Junio, 2019 • 111-126

ISSN 2215-2849 • EISSN: 2215-4752

URL: http://www.revistas.una.ac.cr/index.php/dialogo/index CORREO ELECTRÓNICO: universidadendialogo@una.cr

DOI: https://doi.org/10.15359/udre.9-1.7

las condiciones de salud, la religión, las opiniones, las preferencias sexuales, el estado civil o cualquier otra que atente contra la dignidad humana y tenga por objeto anular o menoscabar los derechos y libertades de las personas." Así pues, encontramos una seria violación a la dignidad humana de las personas con discapacidad, al no encontrar en nuestra institución educativa la verdadera adecuación a sus necesidades principales. La inclusión de las personas con discapacidad no se logra con la simple construcción de rampas y señalamientos de espacios para dichas personas, nuestra Universidad ha tratado, pero no ha logrado la verdadera inclusión de su infraestructura a dicho reto. No se diga si hablamos del personal docente, el cual tampoco está capacitado y mucho menos instruido en este tema en el ámbito de las discapacidades. Nuestra universidad, y consideramos que igualmente todas las de nuestro país, deben velar por lograr la inclusión de las personas con discapacidad.

Palabras clave: inclusión, discapacidad, dignidad, derecho humano, universidad.

Abstract. Even though it is true that our university has been trying to reach inclusion, this is not achieved yet. We are talking about the inclusion of disabled people; notwithstanding that the institution has accepted them, it has not been able to grant them the guarantee of that inclusion, violating with this what the Constitution establishes in its first article, "It is prohibited all kinds of discrimination due to ethnic origin or nationality, gender, age, disabilities, social condition, health conditions, religion, opinions, sexual preferences, marital status, or any other condition that is considered as a violation of human dignity or targets to destroy or reduce people's rights and freedoms." So, we consider a severe violation of human dignity of disabled people, since they do not find true adaptation to their needs in our educative institution. Integrating disabled people is not possible just with signs in the premises and simply building ramps for them. Our university has tried this but has not reached the true inclusion of its infrastructure to this challenge yet. Even more, if we are talking about teachers, they have not been trained and much less educated on this subject related to disabilities. Our university, and we consider that all other universities in our country, should ensure the inclusion of disabled people. "Being different is not the problem; the problem is being treated differently."

Keywords: inclusion, disability, dignity, human rights, university.

Ser diferente no es el problema,el problema es ser tratado diferente

\section{Introducción}

Si bien es cierto que la inclusión educativa requiere mayor atención en los niveles básicos y de educación media superior, no es por ello menos importante 
URL: http://www.revistas.una.ac.cr/index.php/dialogo/index

CORREO ELECTRÓNICO: universidadendialogo@una.cr

DOI: https://doi.org/10.15359/udre.9-1.7

analizarlo en el contexto de la educación superior, como en este caso en nuestra máxima casa de estudios: la Universidad Autónoma de Baja California.

Este trabajo va encaminado a reconocer el esfuerzo y la entereza de aquellas personas que aún con capacidades diferentes o con las discapacidades que poseen luchan cada día por tener un espacio educativo en las mejores universidades de México y del mundo. La Universidad Autónoma de Baja California debe comenzar por tener más apertura en sus espacios para que puedan cursar alguna de las carreras profesionales que oferta la misma Institución.

Nuestro objetivo es que se reconozca la falta de infraestructura en edificios, programas de cómputo, libros y espacios, entre otros, para aquellas personas que necesitan lugares aptos para estudiar, con estrategias y recursos especiales para que puedan lograr un aprendizaje significativo como estudiantes y que el profesor pueda realizar su trabajo adecuadamente.

No negamos, ni demeritamos el esfuerzo que ha pretendido y realizado nuestra universidad para lograr la llamada inclusión educativa, sin embargo, con solo echar una mirada a los edificios y demás espacios y escuchar los reclamos de quienes tienen alguna discapacidad se devela otra realidad. Es decir, la universidad en general está constituida y construida para la gente a la que se le llama normal; es muy triste y denigrante que en pleno siglo XXI a alumnos y maestros que poseen una capacidad diferente o tienen una discapacidad se les dificulte estudiar y trabajar en esta institución, aun cuando nos llamamos una universidad inclusiva.

Somos un país donde se reconocen los derechos humanos, la Universidad Autónoma de Baja California reconoce los derechos humanos a sus trabajadores y a sus alumnos, fomentando y protegiendo ampliamente esos derechos y promoviéndolos en la comunidad universitaria, justo entonces es que por medio de los programas educativos bastos que posee la UABC se integran recursos, técnicas y estrategias encaminadas a que una persona con alguna discapacidad o que posee una capacidad diferente pueda lograr un aprendizaje significativo en su proceso de enseñanza-aprendizaje.

Cierto es que los alumnos requieren esa protección por parte de la UABC, pero también algunos profesores que por la edad o por alguna enfermedad o accidente necesitan apoyos adecuados para realizar su trabajo académico; además se requiere que los profesores en general aprendan a comunicarse con los alumnos con estas características con el fin de obtener el aprendizaje significativo entre ambos. Y sobre todo es necesaria la sensibilización de toda la comunidad universitaria en torno al tema de la no discriminación a través de la inclusión. 
ReVISTA UNIVERSIDAd EN DiÁlogo • Vol. 9, N. ${ }^{\circ}$ 1, Enero-Junio, 2019 • 111-126

ISSN 2215-2849 • EISSN: 2215-4752

URL: http://www.revistas.una.ac.cr/index.php/dialogo/index CORREO ELECTRÓNICO: universidadendialogo@una.cr DOI: https://doi.org/10.15359/udre.9-1.7

\section{Justificación}

Es necesario plantear una propuesta sobre el trato que se da a ciertos sectores de la población universitaria, tanto a profesores como a alumnos, quienes son los que más sufren por no ser tratados como los demás. Desafortunadamente en México no se ha podido desarrollar como se debiera la inclusión de personas con capacidades diferentes o con alguna discapacidad y las instituciones educativas no han sido la excepción, atentando sin tener la intención, contra la dignidad humana.

Es cierto que el artículo primero de la Constitución Política de los Estados Unidos Mexicanos refiere que debemos respetar los derechos humanos, quedando prohibida cualquier forma de discriminación y protegiendo ampliamente a la persona por ser la titular de los derechos y de las obligaciones.

La Universidad Autónoma de Baja California no cuenta con suficientes espacios y estructuras adecuadas, libros, programas de cómputo, entre otras cosas, para aquellas personas, tanto profesores como alumnos, que tienen una discapacidad o presentan una capacidad diferente, por eso es que queremos proponer que se construyan espacios dignos y decorosos para aquellas personas que no ven, no escuchan, no hablan, son pequeñas a comparación de las personas "normales", se trasladan en sillas de ruedas, requieren de un lazarillo para guiarse, de un bastón para apoyarse, son obesas en algunos casos o son adultos mayores. Nuestra universidad en general no cuenta con esos espacios y a las personas con esa condición se les dificulta incluso arribar a la institución, aun cuando en nuestro campus de Tijuana se construyó un puente peatonal, este no se construyó para aquellas personas con condiciones físicas especiales o por lo menos se creyó que se cumplía con ese requisito.

Además, los profesores y alumnos que tienen que utilizar bastones para trasladarse o incluso muletas o sillas de ruedas tienen que subir escaleras porque no existen rampas para ese fin. Lamentablemente en la mayoría de los edificios de nuestra universidad no se cuenta con elevadores, ni rampas suficientes para ese fin, además cuando una persona con alguna capacidad diferente o discapacidad ingresa los profesores no son aptos para instruirla académicamente.

Proponer por medio de las reestructuraciones de edificios, espacios y programas de estudio y de la adquisición de programas de cómputo, de libros digitales, entre otros, el reconocimiento de los derechos a aquellas personas que por su condición no tienen las mismas capacidades físicas que la mayoría que integra la comunidad universitaria es de vital importancia si queremos que realmente a 
URL: http://www.revistas.una.ac.cr/index.php/dialogo/index

CORREO ELECTRÓNICO: universidadendialogo@una.cr

DOI: https://doi.org/10.15359/udre.9-1.7

la $\mathrm{UABC}$ se le considere como una institución educativa incluyente, respetuosa y reconocedora de los derechos fundamentales de los seres humanos, independientemente de su capacidad física, y así reconocer la dignidad humana de todos sus estudiantes, maestros y demás personal que la constituyen.

\section{Desarrollo}

Nuestra Universidad, si bien es cierto que se ha preocupado por dar cabida a todo tipo de personas, es decir, ser inclusiva en la educación que proporciona, se ha quedado rezagada en algunos aspectos, pues la verdad es que no se trata de que permita el ingreso de personas con alguna discapacidad, sino de que sea verdaderamente incluyente, y esto se logra solo satisfaciendo las necesidades de esas minorías.

Es cierto que la Universidad ha hecho lo posible, pero le falta mucho por hacer, de ahí que se diga que la inclusión no ha sido garantizada, faltando con ello a la garantía de ese derecho humano, que pudiera decirse que es la no discriminación.

Como hemos precisado, la universidad no lo ha hecho con intención, por el contrario, ha intentado ser incluyente a través de la donación de computadoras para personas con discapacidad visual, en las que se les incluyen programas de lectores de pantalla para que puedan acceder a la información y así tener una inclusión en el desarrollo de sus estudios profesionales.

Quizá también parte de la falta de ese cumplimiento de la inclusión se deba a que no ha logrado responder a la demanda de las personas con discapacidad y ello deriva del hecho de que esta población no incluye solo a los ciegos y los sordos, sino también a quienes padecen el síndrome de Asperger, personas con retraso mental no severo, personas con parálisis cerebral pero aptos para estudio, personas con tetraplejia, entre otras.

Ahora bien, otro de los grandes retos de la UABC para lograr la inclusión educativa y así garantizarla como un derecho humano, es el hecho de saber cuántos alumnos son los que ingresan, es decir, qué demanda tiene de personas con alguna discapacidad y sobre todo saber cuántos de los alumnos a los que se les da cabida al estudio en cualquiera de las diversas carreras que ofrece nuestra universidad culminan satisfactoriamente sus estudios profesionales, pues este es el parámetro para demostrar fehacientemente que se está cumpliendo con la inclusión educativa.

Debemos entender que ser incluyente también implica que la universidad proporciona el derecho, permite el acceso a la educación, pero al no tratarse 
ReVista Universidad EN Diálogo • Vol. 9, N. ${ }^{\circ}$ 1, Enero-Junio, 2019 • 111-126

ISSN 2215-2849 • EISSN: 2215-4752

URL: http://www.revistas.una.ac.cr/index.php/dialogo/index CORREO ELECTRÓNICO: universidadendialogo@una.cr

DOI: https://doi.org/10.15359/udre.9-1.7

de una institución con educación especial, podría dejar de estar garantizando dicha inclusión y con ello violentando un derecho humano, ya que para que se pudiera decir que cumple plenamente con la inclusión nuestra universidad debería hacer ajustes razonables, basados en las necesidades individuales de cada persona que presente una discapacidad o capacidad disminuida, buscando apoyar en lo necesario para lograr una formación efectiva en lo académico y en lo social, logrando con ello una verdadera integración educativa.

La UABC debe incluir a aquellas personas que se les dificulta estudiar una carrera profesional debido a su condición; no es por medio de un apoyo económico sino por medio de adecuaciones para cada una de las discapacidades físicas y para aquellas personas que tienen una capacidad diferente.

Por ende, el estudiante que aprobó su examen psicométrico y académico debe contar con la seguridad de que la UABC le proveerá no solo edificios y espacios, sino maestros capacitados para atender a este tipo de estudiantes, que los programas de estudios tomen en consideración a este tipo de personas, que los programas digitales están adecuados a las necesidades no solo de las personas con discapacidad visual, sino también de las personas con discapacidad auditiva, entre otras, en otras palabras, deben estar acorde a su capacidad diferente o su discapacidad física.

Sin embargo, nos damos cuenta de que aunque nuestra universidad pretende ser inclusiva, no logra esa realidad, porque, como lo hemos venido refiriendo, no se trata solo de invidentes, o de sordos, cuadripléjicos o cualquier otra discapacidad ya reconocida, debemos hablar también de jóvenes de estatura pequeña (con enanismo), que deben subir escalones para llegar a los salones de clase, personas con sobrepeso que no caben "literalmente" en los mesa-bancos, personas zurdas que no encuentran un mesa-banco adecuado a sus necesidades, porque es evidente que nuestra universidad está hecha para personas llamadas "normales", independientemente de que presenten una discapacidad.

Es tan fácil que una persona se sienta relegada de su calidad de persona porque la sociedad no tiene la cultura del respeto a las personas diferentes; aceptar que la dignidad humana es para los humanos independientemente de las capacidades físicas e intelectuales que se poseen; entonces la misma sociedad aprendería a respetar al otro ser humano por la dignidad misma.

Por ello, como lo refiere Alicia Carolina Cobián Álvarez, invidente, alumna de la Facultad de Derecho de Tijuana de la UABC en su entrevista, que es necesario más que otra cosa, que "se den cursos de sensibilización tanto a 
URL: http://www.revistas.una.ac.cr/index.php/dialogo/index

CORREO ELECTRÓNICO: universidadendialogo@una.cr

DOI: https://doi.org/10.15359/udre.9-1.7

los administrativos, a los maestros y sobre todo a los alumnos", ello con la finalidad de que entiendan que existen personas diferentes a todos los demás, con necesidades especiales o diferentes y que con ello se iniciaría o sería la manera de ir avanzando en la verdadera inclusión, cuando se respete a toda persona con capacidades diferentes o con discapacidades.

La UABC tiene el compromiso de ser ejemplo para las demás instituciones educativas universitarias; aceptando este compromiso la sociedad emularía lo que esta Institución realiza a favor de la comunidad universitaria, y con la garantía de los valores que la UABC se ha preocupado por transmitir, entonces no habría duda de que los profesores y estudiantes con esas capacidades diferentes o con discapacidades físicas estarían más que dispuestos a realizar el proceso de enseñanza-aprendizaje con la calidad que la misma institución merece.

Estas personas necesitan los espacios y las reestructuraciones sobre los edificios, puentes y rampas mismas de la UABC, contribuyendo con la no discriminación hacia estos grupos que pudieran considerarse vulnerables o con condición diferente; la inclusión refiere a que las personas con estas condiciones no se sientan relegados de la misma Institución, al contrario, que ellos perciban que nuestra universidad piensa en este tipo de grupo y quiere fomentar a través de la misma inclusión el respeto hacia la dignidad humana de estas personas especiales, en el buen sentido de la palabra.

Preocuparse por tener instalaciones adecuadas para las personas que laboran y estudian en la $\mathrm{UABC}$, sin hacer distinción alguna, es una muestra de que en la Institución no existe discriminación. Ser incluyente significa que dentro de la preocupación de la universidad están esos grupos vulnerables que requieren apoyo especial para que obtengan la mejor educación universitaria, sin embargo, aun cuando se han hecho esfuerzos para atender a la inclusión no se ha logrado por completo.

Es importante que se entienda que cada una de las personas es vulnerable a convertirse en una persona con alguna discapacidad porque no se tiene el control de los accidentes y de las enfermedades que pueden aparecer de repente en las vidas, por eso, insistimos en que no se trata de compadecer o de compadecerse de estas personas, sino más bien de incluirlas a fin de que estas personas que día a día se enfrentan a los obstáculos de las deplorables estructuras y los nulos espacios que existen para ello, se sientan no solo incluidos, sino un alumno más que ha sido incluido o un profesor o trabajador más que ha sido incluido. 
ReVISTA Universidad EN DiÁlogo • Vol. 9, N. ${ }^{\circ}$ 1, Enero-Junio, 2019 • 111-126

ISSN 2215-2849 • EISSN: 2215-4752

URL: http://www.revistas.una.ac.cr/index.php/dialogo/index CORREO ELECTRÓNICO: universidadendialogo@una.cr

DOI: https://doi.org/10.15359/udre.9-1.7

Con el fin de que nuestra universidad comience a alcanzar esta inclusión, que quizá nunca se logre debido a las diversas discapacidades que se presentan o de las que se sabe día a día, se tendrá que estar en constante reestructuración y es algo que difícilmente se puede hacer y menos lograr.

Si bien es cierto, como lo dijo Alicia Cobián, es importante que las personas con discapacidad se adecuen a lo que actualmente ofrece nuestra institución, pues si bien es cierto que les han dado la oportunidad de estudiar y con ello ya cumplen en parte con la inclusión, pero si en verdad desean cumplir con dicho derecho humano deben entonces adecuar los espacios para que tanto las personas con discapacidad visual o los cuadripléjicos o personas que por una necesidad temporal requieran de utilizar una silla de ruedas, tengan la oportunidad de transitar a través de toda nuestra universidad y de acceder a todas las instalaciones de manera confiable y sin restricción alguna.

Uno de los problemas que presenta nuestra universidad es que a menos que se trate de un estudiante de primero y en ocasiones de segundo semestre, estos no eligen su salón de clases, lo que le facilita a la Dirección de cada unidad académica a proporcionar los espacios necesarios y adecuados para aquellas personas que presentan alguna discapacidad, el problema comienza a partir del tercer semestre, inclusive desde el segundo, en el que el alumno tiene la oportunidad de tomar las materias en los grupos que desee, de ahí que exista la necesidad de los alumnos de estarse trasladando de salón en salón o de edificio a edificio.

Ello en virtud de que los mismos alumnos eligen sus horarios y quizá la materia que matricularon se encuentra en un edificio que carece de rampas o de elevador (casi todos los edificios de nuestra universidad) deben estarse cambiando de salones, por lo que si estos alumnos (que presentan alguna discapacidad) no hacen del conocimiento de la dirección su presencia en dicha materia o edificio, será casi seguro que no se adecue a las necesidades especiales, tratándose principalmente de personas que requieren silla de ruedas o muletas, con lo que impedimos que nuestra institución sea incluyente.

Insistimos en que nuestra universidad es incluyente, pero no logra llegar a serlo en su totalidad y es ahí donde vulnera el derecho humano de esas personas que presentan necesidades especiales, que, como hemos dicho, no solo nos referimos a aquellos que son sordos, ciegos, cuadripléjicos, en silla de ruedas, en muletas, etcétera, sino también a aquellos que presentan algún síndrome, como el de Asperger o que presentan un problema de obesidad y por ello no caben en los mesa-bancos o aquellos que son zurdos, ellos no son del todo considerados por no presentar propiamente una discapacidad, pero a ellos 
URL: http://www.revistas.una.ac.cr/index.php/dialogo/index

CORREO ELECTRÓNICO: universidadendialogo@una.cr

DOI: https://doi.org/10.15359/udre.9-1.7

también les afecta el hecho de que nuestra institución no los tome en cuenta, violentando de nuevo un derecho humano como lo es la no discriminación al no ser totalmente incluyentes.

Cuando se acepten las carencias en la estructura de los edificios y de los espacios construidos vinculados con las condiciones específicas de aquellas personas que poseen capacidades diferentes o discapacidades físicas, ya sea por la misma naturaleza o por algún accidente que sufrió la persona, fundamentalmente se crearía conciencia de las necesidades de modificar los centros educativos para que estas personas tenga el acceso más fácil a sus salones de clases y se pueda lograr ese aprendizaje significativo, en el cual la $\mathrm{UABC}$ ha estado a la vanguardia en cuanto a los métodos de enseñanza, pero que todavía pareciera que los espacios son reducidos para aquellas personas que tienen una capacidad diferente o una incapacidad física.

Entre algunas de las acciones o decisiones que se podrían tomar a corto plazo y que quizá no requerirían de tanta inversión económica y de tiempo serían las de aumentar el número de pupitres adecuados para aquellos que escriben con la mano izquierda, que le añaden un escalón a ciertos pupitres para que las personas pequeñas alcancen a apoyar sus pies; a que algunos pupitres que cuenten con más espacios entre la paleta y la espalda para que puedan ciertas personas con sobrepeso sentirse cómodas y no apretadas. Que las personas que caminen con algún apoyo, como los bastones, muletas o se trasladen en sillas de ruedas, se detecten y se les asigne un salón que se encuentre cerca o el primer piso y así evitar que puedan a llegar a lastimarse por esa situación.

Otra acción inmediata que se puede realizar es la de ofrecer terapia psicológica a toda aquella persona que por capacidad diferente o discapacidad física lo requiera, pues es importante brindar apoyo en este sentido para que el profesor o en su caso el alumno trabaje con su autoestima y no esté en actitud defensiva cuando se toque un tema que puede herir susceptibilidades comunes.

Así mismo, como lo señaló Alicia Carolina Cobián Álvarez, debe darse un curso de sensibilización en principio a alumnos y maestros y también al personal administrativo a fin de ser mas incluyentes con personas con capacidades diferentes, discapacitadas o personas que presentan algún síndrome y que los mismos en ocasiones, por no decir que siempre, no son notorios.

Que los espacios destinados para personas discapacitadas sean los suficientes, tomando en cuenta el número de personas con necesidades especiales matriculadas en cada unidad académica, que se cuente con accesos destinados para 
Revista Universidad EN DiÁlogo • Vol. 9, N. ${ }^{\circ}$ 1, Enero-Junio, 2019 • 111-126

ISSN 2215-2849 • EISSN: 2215-4752

URL: http://www.revistas.una.ac.cr/index.php/dialogo/index CORREO ELECTRÓNICO: universidadendialogo@una.cr

DOI: https://doi.org/10.15359/udre.9-1.7

personas discapacitadas, que las rampas de acceso no solo sean para los proveedores que traen mercancías para los vendedores de la cafetería o para trasladar la basura por parte de las personas de intendencia, se rehagan esas rampas para que tengan un verdadero propósito y no que solo se hagan sin considerar quién o quiénes las utilizarán y puedan trasladarse incluso con la silla de ruedas.

Se pueden hacer las gestiones necesarias en el Municipio o el Gobierno del Estado para que se cuente no solo con un puente peatonal, o bien que se adecue el que ya se encuentra en nuestra institución para que cuente con una rampa verdaderamente apropiada para las personas con discapacidad física, ya sea porque utilizan sillas de ruedas o les resulta complicado subir las escaleras, como algunos de los adultos mayores.

Nuestra Universidad debe tratar a todos por igual; no debe haber un trato diferente de ninguna forma; en algunas ocasiones se puede hacer la excepción por las discapacidades físicas que tiene el alumno, como la visual, por ejemplo que el alumno no pueda realizar un examen por escrito y entonces se deba realizar la prueba de forma oral, pero también darle oportunidad al grupo, con anuencia del director de la Facultad que corresponda, para que si alguno quiere realizar examen de esa forma también lo presente, no importando si es mensual o semestral, incluso extraordinario, porque si solamente es para aquellas personas con discapacidad o con capacidades diferentes, entonces, también caemos en la discriminación y con ello en el trato diferente a los diferentes.

Difícil es para el profesor no hacer diferencia, difícil es impartir las clases por el número de alumnos que se tienen, más cuando existe entre ese grupo una persona con alguna discapacidad o capacidad diferente, por lo que se pretende que el profesor universitario esté más que dispuesto a capacitarse en ese rubro, y si alguna vez le tocara ser profesor de un sordo, por ejemplo, permitir el apoyo de un intérprete o capacitar a los profesores para que ellos mismos realicen esta actividad.

De ahí que, para que nuestra Universidad Autónoma de Baja California sea realmente incluyente o vaya encaminada a ese fin, puede realizar como hemos señalado acciones que no son del todo costosas y que no requieren mucho esfuerzo, pero sí de bastante participación activa por parte de las autoridades de la institución y de las unidades académicas.

La universidad debe, a través de su escuela de idiomas, proporcionar cursos didácticos encaminados a enseñar a los maestros el lenguaje de señas y por parte de la Facultad de Medicina y Psicología proporcionar cursos de sensibilización, 
URL: http://www.revistas.una.ac.cr/index.php/dialogo/index

CORREO ELECTRÓNICO: universidadendialogo@una.cr

DOI: https://doi.org/10.15359/udre.9-1.7

así como impartir cursos en los que se dé a conocer las diversas limitaciones que pueden presentar los alumnos y cómo atender a esas limitaciones o cómo atender o tratar a personas con capacidades diferentes, e incluso cómo atender o impartir clases a alumnos que presenten algún síndrome o enfermedad, que implique algún trato especial en clase.

Hay mucho que hacer en este rubro: la Universidad Autónoma de Baja California junto con sus profesores y alumnos deben ser más empáticos en este tipo de situaciones, desde que se presenta un alumno con estatura pequeña hasta una persona que tiene condición de zurdo, los invidentes, las personas con sillas de ruedas porque tienen parálisis motriz o aquellos que por alguna situación traen muletas o incluso bastones. Cualquiera puede alguna vez estar en esta situación y es cuando se toma conciencia de lo difícil que resulta el trasladarse de un lugar a otro en la UABC, y cuando se es zurdo y los mesa-bancos y todas las herramientas se hicieron para los diestros es cuando entendemos que se requiere una verdadera restructuración.

Ya lo señala J. Vidal-Bota en su ensayo La dignidad humana y sus implicaciones éticas: "La dignidad humana es el derecho que tiene cada ser humano, de ser respetado y valorado como ser individual y social, con sus características y condiciones particulares, por el solo hecho de ser persona", es decir, una persona por el solo hecho de serlo debe ser respetada y se le deben reconocer sus derechos y obligaciones. El respeto es precisamente considerar sus capacidades diferentes o discapacidades físicas para no excluirlos de un derecho humano como la educación, según el artículo $3^{\text {ro }}$ constitucional.

Insistimos en que nuestra Universidad ha tratado de ser incluyente, pero no lo ha logrado a plenitud, de ahí que se haga énfasis en que la misma no garantiza aún el derecho humano de la inclusión educativa, como también lo refirió Alicia Cobián, alumna de la Facultad de Derecho de Tijuana, al referirnos que al ingresar a nuestra Facultad le hubiera gustado que el director o cualquiera de las autoridades universitarias o de la propia unidad académica le hubiera dado un recorrido por nuestra Facultad, ahora su espacio de estudio, para haberse podido ubicar y así localizar en dónde quedaba cada lugar, en específico se refería a la Dirección. Ella misma manifiesta que le gustaría que los alumnos y maestros, así como las personas con las que interactúa, tomaran conciencia de que los espacios como escaleras, pasillos, rampas y demás deben estar libres, y no encontrarse con personas detenidas sentadas en las escaleras, personas sin ninguna discapacidad utilizando las rampas, pues para ella es más fácil la utilización de las rampas para trasladarse, ya que de lo contrario debería estarse deteniendo en cada cambio de banquetas. 
Revista Universidad EN DiÁlogo • Vol. 9, N. ${ }^{\circ}$ 1, Enero-Junio, 2019 • 111-126

ISSN 2215-2849 • EISSN: 2215-4752

URL: http://www.revistas.una.ac.cr/index.php/dialogo/index CORREO ELECTRÓNICO: universidadendialogo@una.cr

DOI: https://doi.org/10.15359/udre.9-1.7

Nos refirió además que le gustaría que hubiera señalamiento en los salones en el lenguaje o señalamiento braille para así lograr una más pronta ubicación de los salones. También señalaba que habría de contarse con medios para mejor ubicación y localización de las otras unidades académicas o de la Vicerrectoría y que ello se lograría poniendo anuncios escritos en braille, o bien que tuvieran estantes con algún sistema de sonido para mayor localización.

Alicia Carolina Cobián Álvarez como estudiante de la Facultad de Derecho tuvo una participación peculiar, encaminada a demostrar que se puede lograr implementar recursos para que los invidentes como ella puedan acceder a los libros que se ocupan para el estudio en la Universidad, y fue así como participó en la elaboración en braille de la constitución para niños, a fin de acercar recursos didácticos y de estudio para niños que sufren su misma discapacidad.

Así pues, nuestra universidad debería invertir en la traslación al braille de algunos libros, por lo menos los que son más solicitados por los maestros, para que así puedan acceder a estos recursos didácticos todos aquellos estudiantes que sean ciegos, con ello se estaría iniciando una verdadera inclusión educativa.

Otro aspecto que la alumna entrevistada nos manifestó fue el hecho de que si bien es cierto que la UABC abre sus puertas a personas con discapacidad, no cumple cabalmente con la inclusión, pues no toma en cuenta que sus portales, de los cuales se obtiene información para seleccionar horarios, para registrarse en el servicio social, ya sea comunitario o profesional, y para inscribirse en las prácticas profesionales no pueden ser leídos por los programas que utilizan los invidentes para ese fin, es decir que los lectores de pantalla que utilizan no pueden leer la información, quedando de nuevo de manifiesto que la inclusión educativa no solo se trata de dar cabida y matricular alumnos con discapacidad, sino que ello implica mucho más.

Pero no todo está perdido, pues hemos encontrado profesores y profesoras de diferentes facultades, en específico de la de Humanidades, que han coordinado con el Departamento Psicopedagógico de la Facultad para que los oriente sobre cómo tratar a las personas que presenten alguna discapacidad o enfermedad mental.

En esta misma Facultad algunas alumnas con discapacidad auditiva tomaron la iniciativa de buscarse maestros que supieran el lenguaje de señas y que les sirvieran de intérpretes en sus clases, logrando así de manera personal obtener recursos para lograr un aprendizaje significativo, sin que ello haya sido proporcionado por la propia Universidad. De ahí que, como nos refería Alicia Cobián, es necesario también que las personas con discapacidad busquen sus propios recursos para salir adelante. 
URL: http://www.revistas.una.ac.cr/index.php/dialogo/index

CORREO ELECTRÓNICO: universidadendialogo@una.cr

DOI: https://doi.org/10.15359/udre.9-1.7

Así lo refirió la psicóloga Jany Ramírez al manifestarnos en una entrevista con ella que, a título personal y dada la inquietud de algunos maestros, realizó algunas conferencias de concientización relacionadas con los temas de las discapacidades y de las diversas enfermedades mentales. Así mismo, invitó a un psicólogo experto en la materia para que enseñara a los maestros cómo tratar a personas ciegas, específicamente.

Además, a petición de algunos alumnos sordos, consiguió para su Facultad de Humanidades, campus Tijuana, intérpretes, logrando con ello una verdadera escuela inclusiva, haciendo que dichas personas se sientan seguras, acogidas, pero sobre todo que se sientan valoradas.

Nos refería también que su trabajo por la inclusión educativa no ha terminado ahí, pues ahora se encuentra trabajando con otros profesores a fin de especializarse para estar en aptitudes de impartir clases a personas con autismo, con síndrome de Asperger y de otras enfermedades mentales.

A estas acciones se les llama inclusión, nuestra universidad debe voltear a mirar a estas personas que pertenecen a su misma comunidad universitaria y solicitarles el apoyo para que sean multiplicadores de sus conocimientos y se convierta en un proyecto institucional a fin de que toda la Universidad Autónoma de Baja California cuente con personal docente capacitado para enfrentarse a los retos de una verdadera inclusión educativa.

Insistimos en que si bien es cierto que nuestra institución universitaria pugna por ser incluyente y que lo hace a través del Comité de Equidad, el cual se encarga de velar por quienes se encuentran en situaciones menos favorables, para que tengan acceso a una formación profesional con la calidad que brinda la UABC, sin embargo, insistimos en que, como lo refirió Alicia Cobián, no hay libros en braille en la biblioteca central, no existen los mismos en digital como para que los puedan leer sus lectores de pantalla. Los centros de cómputo, tanto el central como los de cada unidad académica, no cuentan con los lectores de pantalla, por lo que los estudiantes deben traer sus computadoras portátiles para poder realizar sus tareas.

Si bien es cierto que el rector de nuestra máxima casa de estudios, el doctor Juan Manuel Ocegueda Hernández, hizo entrega de computadoras portátiles a personas con discapacidad, específicamente a personas ciegas, también es cierto que dicho recurso no llegó a todos los estudiantes, pues fue otorgado una sola vez, por lo que aquellos que habían interrumpido sus estudios o aquellos que acaban de iniciar sus estudios después de esa entrega no fueron beneficiados con dichos recursos, de ahí que insistimos en que aún cuando 
la UABC ha intentado la inclusión educativa, no lo ha logrado todavía, violentando así un derecho humano.

Pugnemos entonces por una universidad incluyente y reconocedora de los derechos humanos, pero sobre todo reconocedora de la dignidad humana.

\section{Conclusiones}

La Universidad Autónoma de Baja California debe ofertar cursos para docentes de concientización, del lenguaje de señas, cursos sobre cómo tratar e impartir clases a personas con algún síndrome, autistas o con alguna enfermedad mental.

Debe contratarse maestros capacitados o especializados en las diversas discapacidades, sobre todo tomando en cuenta la demanda que presenta la universidad en este rubro de las discapacidades, quienes en su momento se podrán convertir en capacitadores o multiplicadores, en pro de la inclusión educativa.

Deben sobre todo adecuarse los espacios de estudio, la infraestructura total de la Universidad, adecuarla a las necesidades de una minoría, pero que refleja la totalidad de la inclusión educativa.

También deben adecuarse los programas de cómputo de los portales de las páginas de la Universidad a fin de que todos tengan acceso a la información que ahí se les proporciona y así lograr ser más inclusivos.

Es fundamental invertir en la adquisición de libros digitales o adaptados al braille para que los que tengan una discapacidad visual, tengan acceso a la información bibliográfica y con ello propiciar que dichas personas se sientan seguras, acogidas, pero sobre todo que se sientan valoradas e incluidas.

Asimismo, es necesario establecer como una medida institucional dar cursos sobre sensibilización a todo el personal administrativo a fin de que se logre dar, como hasta ahora, un trato digno a las personas que presenten alguna discapacidad.

Demandar de las autoridades tanto federales como estatales el apoyo económico para la adquisición de libros, sistemas de cómputo, señalamientos en braille, modificar la infraestructura, la construcción de espacios destinados a personas con discapacidades, la instalación de elevadores, demostrando para ello las carencias que existen para aquellas personas que tienen una limitante en su capacidad física o tienen una capacidad diferente. 
Que se entreviste a los alumnos que sufren de alguna discapacidad o tengan algún síndrome y que se matriculen en las diversas unidades académicas de la Universidad Autónoma de Baja California a fin de conocer sus necesidades, ya que, tratándose de una minoría, requieren una atención especial, misma que se podría atender si se conocen de inicio sus necesidades particulares.

Que en el Plan de Desarrollo Institucional de los futuros rectores se prevea la verdadera inclusión educativa a fin de garantizarles que no se sientan discriminados y con ello garantizar verdaderamente sus derechos humanos.

Lo que se busca finalmente para no violentar los derechos humanos de los discapacitados y así ser una verdadera universidad incluyente es lograr ser una institución en la que no se marquen las diferencias, en la que a esos grupos vulnerables se les otorgue, al igual que a los demás, una educación universitaria de calidad y con calidez.

\section{Referencias consultadas}

Cardona Echaury, Angélica Leticia, Lourdes Margarita Arambula Godoy, Gabriela María Vallarta Santos. Estrategias de atención para las diferentes capacidades. Trillas. Mexico. 2012.

Concepto de dignidad humana. Recuperado de http://deconceptos.com/ ciencias-jurídicas/dignidad-humana

Convención relativa a la Lucha contra las Discriminaciones en la esfera de la Enseñanza. 1960. Recuperado de: http://portal.unesco.org/es/ev.php-

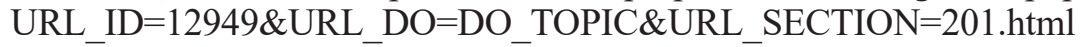

Convención sobre los Derechos del Niño. 1989. Recuperado de: http://www. ohchr.org/SP/ProfessionalInterest/Pages/CRC.aspx

Convención Internacional sobre los Derechos de las personas con Discapacidad. 2006. Recuperado de: http://www.un.org/esa/socdev/ enable/documents/tccconvs.pdf

http://en.calameo.com/read/002155013e6d4e840fdf4

https://www.sep.gob.mx/work/models/sep1/Resource/558c2c24-0b12-4676ad90-8ab78086b184/ley_general_inclusion_personas_discapacidad.pdf

http://www.diputados.gob.mx/LeyesBiblio/regley/Reg_LGIPD.pdf 
http://www.dof.gob.mx//avisos/2517/SEP_010616_01/SEP_010616_01.html

http://www.seslp.gob.mx/pdf/taller2011-2012/uno/DOCUMENTOS/ Inclusion\%20Educativa.pdf

Declaración Universal de Derechos Humanos. 1948 (Artículo 26) Recuperado de: http://www.un.org/es/universal-declaration-human-rights/

Directrices sobre políticas de inclusión en la educación. Recuperado de: http:// unesdoc.unesco.org/images/0017/001778/177849s.pdf

J. Vidal-Bota (2019). Valores y principios. La dignidad humana y sus implicaciones éticas. Recuperado de http://www.ace.org/v_pp.htm

\section{Legislaciones}

Constitución Política de los Estados Unidos Mexicanos

Ley de Atención y Protección a Personas con Autismo para el Estado de Baja California. 2016. (Artículo 8).

Ley de Educación del Estado de Baja California. 1995.

Ley General para la Inclusión de las Personas con Discapacidad. 2011.

Ley para las Personas con Discapacidad en el Estado de Baja California. 2010.

Plan Institucional de Desarrollo de la Universidad Autónoma de Baja California. 2015-2019. 